\title{
Vom Versuch der Rückwärtsrolle des Staates: Verteilungspolitische Aspekte der Finanzpolitik im Konjunkturverlauf
}

Im vergangenen Jahrzehnt stand die Finanzpolitik unter erheblichem Konsolidierungsdruck. Zum einen hatte sie sich diesen Druck selbst auferlegt, indem privaten Haushalten und Unternehmen enorme Steuerentlastungen gewährt wurden. Zum anderen musste sie infolge der anhaltenden wirtschaftlichen Stagnation hohe Steuerausfälle hinnehmen. Die europäischen Verschuldungsgrenzen zwangen zu einer restriktiven Ausgabenpolitik. Unter diesen Bedingungen war es nicht Ziel der deutschen Finanzpolitik, die aus der Entwicklung der Markteinkommen resultierende verteilungspolitische Schieflage zu korrigieren. Damit hat die Finanzpolitik zwei originäre Aufgaben, nämlich die Stabilisierungsaufgabe und die Verteilungsaufgabe, sträflich vernachlässigt.

\section{Einleitung}

Im letzten Jahrzehnt hat die Finanzpolitik eine dominante Rolle in den politischen Auseinandersetzungen gespielt. Mehrere Ereignisse waren hierfür ausschlaggebend. An prominenter Stelle stehen die fiskalischen Belastungen im Zuge des Vereinigungsprozesses. Eine Zäsur bedeutete der Vertrag von Maastricht, der die öffentlichen Haushalte unter erheblichen Konsolidierungsdruck setzte. Zwar erleichterte der Konjunkturaufschwung Ende der 1990er Jahre dieses Projekt, doch wurde die Aufschwungs-„Dividende“ zur Finanzierung umfangreicher Steuersenkungen anstatt zur Budgetkonsolidierung verwendet. Trotz dieser Steuerentlastungen geriet die deutsche Wirtschaft in eine hartnäckige Stagnation, die neuerlich die öffentlichen Haushalte in die Verschuldung trieb. Verteilungspolitisch und gesamtwirtschaftlich höchst problematische Ausgabekürzungen waren die Folge. Zu diesem Zeitpunkt fühlten sich die finanzpolitischen Entscheidungsträger längst nicht mehr einer Politik verpflichtet, die mit ihren Mitteln das Ziel eines gesamtwirtschaftlichen Gleichgewichts anstrebt, wie dies im Stabilitäts- und Wachstumsgesetz von 1967 kodifiziert worden war. ${ }^{1}$ Im Gegenteil - fast gebetsmühlenartig wurde die angeblich wachstumshemmende Ausdehnung des Staatssektors (der Staatsverschuldung) kritisiert und die Ineffizienz staatlicher Prozesspolitik behauptet.

\section{Der Vertrag von Maastricht - Vollzug eines Paradigmenwechsels}

Vor mehr als einem Jahrzehnt waren der Vertrag von Maastricht und in seinem Gefolge der Europäische Stabilitäts- und Wachstumspakt in Kraft getreten. Damit hatte der Paradigmenwechsel der Finanzpolitik hin zu neoklassischem (angebotspolitischen) Handeln seine institutionelle Absicherung gefunden. Nicht zuletzt durch die dort eingezogenen staatlichen Verschuldungsgrenzen wurden die Möglichkeiten des Staates für eine antizyklische Politik stark eingeengt. Doch ging es bei dieser Operation nicht allein um eine Beschneidung der konjunkturellen Eingriffsmöglichkeiten des Staates. Faktisch wurden die Vorkehrungen für eine Neuverteilung der wirtschaftspolitischen Rollen und Machtverhältnisse getroffen, die darauf zielte, den staatlichen Einfluss auf die wirtschaftliche Entwicklung zurückzudrängen.

In der neoklassischen (angebotspolitischen) Sicht sind es die Tarifparteien, die über die Höhe der Beschäftigung entscheiden, während die Zentralbank die Höhe der Inflationsrate bestimmt. Der Staat solle sich darauf beschränken, zur optimalen Allokation der Ressourcen beizutragen und Rechtssicherheit und Ordnung zu garantieren. Bei der Annahme rationaler Erwartungsbildung bleibt systematische Makropolitik, also auch finanzpolitische Stabilisierung, ohne Einfluss auf Produktion und Beschäftigung. Makropolitik ist aus dieser Sicht nicht erforderlich, weil die Hypothe- se ständiger Räumung der Märkte durch flexible Preise zugrunde gelegt wird. Neoklassisches Denken fußt auf der Vorstellung funktionierender Märkte, die durch „exogene" Staatseingriffe eher gestört werden. Staatliche Politik solle sich darauf konzentrieren, für niedrige Steuersätze zu sorgen. Das hoch entwickelte Umverteilungssystem sowie eine umfangreiche Regulierungsbürokratie, so heißt es, erforderten eine zu hohe Abgabenbelastung, die eine erlahmende Wachstums- und Beschäftigungsdynamik zur Folge habe. Ein großzügiger Wohlfahrtsstaat vermindere die Anreize zu Arbeit und Leistung. Hingegen fördere ein niedriger Staatsanteil das wirtschaftliche Wachstum, weil mehr produktive Ressourcen in die nicht-staatlichen Verwendungsbereiche fließen könnten. Erst recht gelte dies in einer globalisierten Welt.

Dieser Sichtweise steht das "traditionelle" europäische Sozialmodell gegenüber, demzufolge staatliche Eingriffe in das

\footnotetext{
1 Mit diesem Gesetz sollte dokumentiert werden, dass sich der Staat seiner Verantwortung für die gesamtwirtschaftliche Entwicklung nicht entziehen könne, sondern eine aktive Rolle zu übernehmen habe (Vesper 2007, S.5ff.).
}

Dieter Vesper, Dr., von 1973 bis 2007 Wissenschaftler im Deutschen Institut für Wirtschaftsforschung (DIW) Berlin. Arbeitsschwerpunkte: Grundsatzfragen der Finanzpolitik, insbesondere finanzpolitische Stabilisierungsfähigkeit gesamtwirtschaftlicher Prozesse, Schuldenpolitik des Staates, der Länder und Kommunen sowie Steuerschätzungen. e-mail:dvesper@diw.de 
Wirtschaftsgeschehen notwendige Voraussetzung für eine sozial abgesicherte Marktwirtschaft sind. Die Aufgaben der Finanzpolitik bestehen demnach vor allem darin,

- öffentliche Güter bereitzustellen;

- die aus dem Marktprozess resultierende Einkommensverteilung zu korrigieren; - für die Absicherung existenzieller Risiken wie Alter, Krankheit oder Arbeitslosigkeit zu sorgen, weil hier individuelles Entscheidungsversagen bei subjektiver Unsicherheit droht (Matzner 1982);

- die inhärente Instabilität marktwirtschaftlicher Entwicklung zu stabilisieren: Die Märkte sind störanfällig, weil Investitionsentscheidungen unter Unsicherheit (unvollständige Informationen, unsichere Erwartungen) getroffen werden, und deshalb staatlicher Interventionen bedürfen.

Im Gegensatz zum neoliberalen Staatsverständnis steht in dieser Sichtweise staatliches Handeln nicht in Konkurrenz zum Markt, sondern in einer Komplementaritäts- und Substitutionsbeziehung. So sichert eine ausreichende Versorgung mit Infrastrukturgütern wie z. B. im Bildungsund im Verkehrssektor die Basis für mehr Wirtschaftswachstum und Wohlstand; diese Güter fördern einmal Rentabilität und Produktivität der privaten Wirtschaft, darüber hinaus stiften sie vielfältigen Nutzen für die privaten Haushalte. Eine Marktlösung würde eine Tendenz zur Unterversorgung sowie eine ungleichgewichtige Verteilung dieser Güter provozieren.

Eine herausgehobene Rolle spielt die staatliche Umverteilungspolitik, auch wenn man nach einem konkreten Verteilungsziel wird suchen müssen. Ein solches Ziel ließe sich schon deshalb nicht definieren, weil jede staatliche Aktivität verteilungspolitische Implikationen nach sich zieht. Es geht nicht nur um die Frage, welche Auswirkungen eine Maßnahme auf die Verteilung der Einkommen oder Vermögen hat; auch Fragen wie der Zugang zum Bildungssystem und/ oder die Versorgung mit Gesundheitsdienstleistungen sind von zentraler Bedeutung. Stets begünstigt oder belastet die Finanzpolitik bestimmte Personen oder Personengruppen: Wer profitiert und wer verliert? Im Übrigen hängt auch die Einstellung bestimmter Personengruppen zu den unterschiedlichen wirtschaftspolitischen Konzepten davon ab, welche Vorteile oder Nachteile erwartet werden. Ansätze, die Staatseingriffe als Störung der markt- wirtschaftlichen Entwicklungs- und Anpassungsprozesse ansehen, werden eher von den sogenannten Wirtschaftseliten (Unternehmer, Manager) präferiert, während Arbeitnehmer bzw. ihre Interessenvertretungen das „Marktversagen“ spüren und deshalb mehr auf Konzepte vertrauen, die Beschäftigung und sozialen Schutz versprechen. $^{2}$

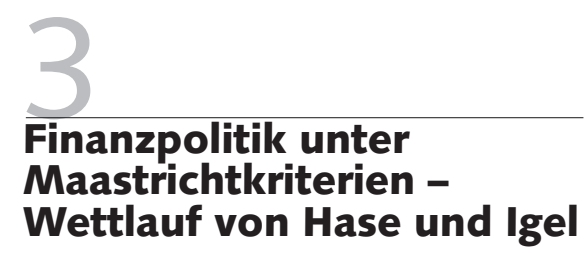

Ein Blick zurück auf das letzte Jahrzehnt zeigt, dass die Finanzpolitik wenig zur Stabilisierung der gesamtwirtschaftlichen Prozesse beigetragen, das Angebot an öffentlichen Gütern zurückgeschraubt und eine Umverteilungspolitik betrieben hat, welche die Bezieher niedriger Einkommen geschwächt und die Bezieher höherer Einkommen gestärkt hat. Die staatlichen Entscheidungsträger folgten einem Konzept, das eindeutig angebotspolitischen Mustern entsprach (vgl. hierzu auch Bofinger 2008).

Mit einem finanzpolitischen Kraftakt und einer Reihe „temporärer" Maßnahmen, die auch Budget-Kosmetik einschlossen, war es 1997 der Finanzpolitik gelungen, die fiskalischen Konvergenzkriterien für die Europäische Währungsunion zu erfüllen und das Staatsdefizit unter die 3-\%Marke zu drücken. Trotz der finanzpolitischen Restriktion geriet Deutschland in den Sog des weltwirtschaftlichen Aufschwungs, und das Staatsdefizit konnte bis zum Jahre 2000 auf $1,1 \%$ des nominalen Bruttoinlandsprodukts (BIP) verringert werden. ${ }^{3}$ Infolge der günstigen Entwicklung der Staatsfinanzen sah die Bundesregierung die Zeit für massive Steuerentlastungen der privaten Haushalte und der Unternehmen gekommen. Die Entlastungen bei der Einkommensteuer summierten sich insgesamt, also nach Inkrafttreten aller Reformstufen, auf über $40 \mathrm{Mrd}$. $€$ jährlich; dies entsprach knapp $2 \%$ des nominalen BIP. Die Unternehmensteuerreform schlug nochmals mit fast $10 \mathrm{Mrd}$ € zu Buche.

Trotz der Steuersenkungen, die stufenweise in Kraft traten, geriet die deutsche Wirtschaft 2001 in eine Rezession, aus der sie sich in den folgenden Jahren nicht lösen konnte. In dieser langen Stagnationsphase stieg die Arbeitslosigkeit auf Rekordhöhen, die zusätzlichen Belastungen für die öffentlichen Haushalte waren enorm. Alles in allem ist das Staatsdefizit bis 2003 auf $4 \%$ des BIP gestiegen und konnte auch danach trotz einschneidender Sparmaßnahmen nur langsam abgebaut werden. Eine Wende zum Besseren trat erst 2006 ein, als im Zuge der konjunkturellen Wende und überraschend hoher Steuereinnahmen die Defizitquote auf 1,7 \% zurückging. Ende 2007 konnte sogar - erstmals seit fast 20 Jahren ein geringes Finanzierungsplus in den öffentlichen Haushalten vermeldet werden. In diesem Aufschwung entwickelten sich die öffentlichen Haushalte fast lehrbuchmäßig antizyklisch: Schnellen in wirtschaftlich schwierigen Zeiten die Staatsdefizite ,quasiautomatisch" in die Höhe, so werden sie in einem Aufschwung ebenso „quasiautomatisch" abgebaut. Ein Teil des Konsolidierungserfolgs ist allerdings der drastischen Erhöhung der Mehrwertsteuer 2007 zuzurechnen. Überschlägig ergeben sich für 2007 aus dieser Erhöhung Mehreinnahmen von rund 19 Mrd. €, d.h. die Defizitposition hat sich allein durch diese Maßnahme um $0,8 \%$ des nominalen BIP verbessert.

Eine Würdigung der konjunkturellen Ausrichtung der Finanzpolitik in den Jahren 2001 bis 2005 kommt zu einem negativen Urteil. In dieser Zeit schöpfte die Finanzpolitik ihr Stabilisierungspotenzial nicht aus: Sie setzte sich zu ehrgeizige Defizitziele und versuchte in einem schwierigen konjunkturellen Umfeld, Einnahmeausfälle durch Ausgabekürzungen auszugleichen - das Ergebnis ähnelte dem Wettlauf von Hase und Igel. Zudem „verschenkte“ sie konjunkturelle Manövriermasse, indem umfangreiche Steuerentlastungen gewährt wurden.

Wenn ein nicht unbedeutender Teil der steuerlichen Entlastungen in die Ersparnisse geflossen ist, so lag dies auch daran, dass

\footnotetext{
Nach Rothschild entsprechen Theorien, welche die Leistungsfähigkeit des Marktes hervorheben, den ideologischen ("natürlichen“) Bedürfnissen der Wirtschaftseliten eher als Konzepte, die eine Instabilität marktwirtschaftlicher Prozesse behaupten. Arbeitnehmer hingegen nehmen das wirtschaftliche Geschehen hin, zumal sie vielfach unbefriedigende Einkommensverhältnisse und das Risiko, arbeitslos oder krank zu werden, vorfinden (Rothschild 1986, S.112ff.).

3 Tatsächlich verbuchten die öffentlichen Haushalte einen Finanzierungsüberschuss, doch enthielt dieser die sog. UMTS-Erlöse in Höhe von rund 50 Mrd. €.
} 


\begin{tabular}{|c|c|c|}
\hline & Einnahmen & Ausgaben \\
\hline 1997 & 45,7 & 48,4 \\
\hline 1998 & 45,9 & 48,0 \\
\hline 1999 & 46,6 & 48,1 \\
\hline 2000 & 46,4 & 45,1 \\
\hline 2001 & 44,7 & 47,6 \\
\hline 2002 & 44,4 & 48,1 \\
\hline 2003 & 44,5 & 48,5 \\
\hline 2004 & 43,3 & 47,1 \\
\hline 2005 & 43,5 & 46,9 \\
\hline 2006 & 43,8 & 45,4 \\
\hline 2007 & 43,9 & 43,9 \\
\hline $\begin{array}{l}\text { Quelle: } \\
\text { Bundes }\end{array}$ & & EILUN \\
\hline
\end{tabular}

in dieser Zeit eine heftige Debatte um die Zukunfts- und Tragfähigkeit des deutschen Rentensystems tobte und in der Einführung der kapitalgedeckten „Riester-Rente“ mündete. Im Ergebnis ist in all den Jahren die Sparquote der privaten Haushalte exorbitant gestiegen, von 9,2\% im Jahre 2000 auf $10,9 \%$ im Jahre 2007. ${ }^{4}$ Auch von der Senkung der Unternehmenssteuern ging zumindest kurzfristig kein merklich positiver Effekt auf die Investitionstätigkeit aus. Höhere Gewinne nach Steuern ziehen nicht automatisch höhere Investitionen nach sich, nämlich dann nicht, wenn die Absatzerwartungen der Unternehmen von Pessimismus geprägt sind oder auch die Abschreibungsbedingungen - wie geschehen - verschlechtert wurden. In früheren Jahren hatte die Kombination von hohen Steuersätzen und günstigen Abschreibungsbedingungen quasi als Investitionspeitsche gewirkt.

Alles in allem ist im vergangenen Jahrzehnt der Anteil des Staates am Bruttoinlandsprodukt kleiner geworden. Während die Einnahmen von $45,7 \%$ auf $43,9 \%$ gesunken sind, verzeichneten die Ausgaben einen Rückgang um immerhin 4,5 Prozentpunkte auf $43,9 \%$. Dies war der niedrigste Wert seit der Wiedervereinigung (Tabelle 1).

\section{Der Rückbau des Sozialstaates}

\section{1 ÖFFENTLICHE GÜTER}

Der Versuch, die von Maastricht gezogenen Defizitgrenzen einzuhalten und gleichzeitig die groß dimensionierten Steuerrefor- men zu finanzieren, zog zwangsläufig Kürzungen auf der Ausgabenseite nach sich. Zunächst wurde der Rotstift bei den öffentlichen Investitionsausgaben angesetzt, die noch die flexibelste Ausgabeart in den öffentlichen Haushalten sind. Anders als die meisten Ausgaben ist die Handlungsfreiheit der politischen Entscheidungsträger bei der Veranschlagung der Investitionen relativ hoch, da es sich hierbei um „freiwillige“ Aufgaben und nicht um Aufgaben handelt, die durch gesetzliche Vorschriften mehr oder weniger festgelegt sind. Im vergangenen Jahrzehnt sind die öffentlichen Investitionsausgaben um ein Fünftel gesunken. Mit einem Anteil von nur noch 1,4 \% am BIP haben sie ein besorgniserregend niedriges Niveau erreicht, auch wenn Sonderfaktoren die Entwicklung verzerrten. ${ }^{5}$ Vor allem waren es die Kommunen als Hauptinvestor der öffentlichen Hand, die ihre Investitionsausgaben zurückschraubten. Ihnen bleibt es infolge der haushaltsrechtlichen Vorschriften in Krisenzeiten verwehrt, Investitionsausgaben über Kredite zu finanzieren, sodass sie $\mathrm{zu}$ prozyklischem Investitionsverhalten gezwungen sind. Verstärkt wird dieser Effekt dadurch, dass die Zuweisungen aus dem kommunalen Finanzausgleich an die Steuereinnahmen der Länder gekoppelt sind und besonders die investiven $\mathrm{Zu}$ weisungen starken diskretionären, von der Haushaltslage der Länder abhängigen Schwankungen unterliegen. Die Abhängigkeit der öffentlichen Investitionstätigkeit von der Finanzlage zeigt sich - nun mit umgekehrten Vorzeichen - in den Jahren 2006 und 2007, die durch einen erfreulichen Zuwachs geprägt waren.

Mindestens ebenso gravierend waren die Einschnitte im Bildungssektor, dessen Bedeutung für die wirtschaftliche Entwicklung inzwischen unübersehbar ist und der deswegen eine wichtige Rolle in den neueren Ansätzen der Wachstumstheorie spielt. Bildungsausgaben erhöhen nicht nur das Produktivitätsniveau und ermöglichen höhere Einkommen; sie sind zudem Voraussetzung für die Innovationsfähigkeit einer Volkswirtschaft und somit für einen höheren Wachstumspfad (Schettkat 2002).

Für den Einzelnen ist der Zugang zum Bildungssystem die Voraussetzung für die Integration in die Gesellschaft und die Erzielung eines hinreichenden Erwerbseinkommens. Es ist die viel zitierte Chancengleichheit, die ihre Konkretisierung in der Frage des Zugangs zum Bildungssystem findet. Gerade Kinder aus Unterschichten sind auf ein funktionsfähiges und gut ausgestattetes Bildungssystem angewiesen, wie die internationalen Vergleichsstudien PISA, IGLU oder TIMMS zeigen (vgl. auch Böttcher 2005). Doch hängt in keinem anderen Land der Welt der Schulerfolg von Kindern so stark von Einkommen und Bildung der Eltern ab wie in Deutschland, dessen Schulsystem offenbar nicht in der Lage ist, gute Lernergebnisse unabhängig von der sozialen Herkunft zu generieren (vgl. u.a. Allmendinger/Helbig 2008). Beispielsweise sind Ganztagsschulen in vielen EU-Ländern der Normalfall, in Deutschland besteht hingegen ein eklatanter Mangel, der darauf hindeutet, dass zu wenig Geld in den Ausbau investiert wird. An den Schulen ist der Personaleinsatz rückläufig, und das überalterte Lehrpersonal wird nicht ausreichend ersetzt. Auch das Angebot an Kinderbetreuungseinrichtungen ist, insbesondere in Westdeutschland, höchst lückenhaft. Ebenso fordert das System der Hochschulausbildung Kritik heraus. Neben der Qualität der Ausbildung wird die geringe Beteiligung an höheren Bildungsgängen kritisiert.

All dies nährt die Vermutung, dass die Politik die rückläufigen Schülerzahlen im Bildungsbereich für eine finanzielle Entlastung der öffentlichen Haushalte genutzt und weniger für günstigere Betreuungsrelationen gesorgt hat. Einer OECD-Untersuchung zufolge sind die Bildungsausgaben in Deutschland im vergangenen Jahrzehnt in wesentlich geringerem Maße gestiegen als im OECD-Mittel (OECD 2007); auch war der Anteil der Bildungsausgaben am BIP mit 3,5 \% deutlich niedriger als im Durchschnitt der OECD-Länder (5,3\%). So schneidet Deutschland beim Vergleich der Schüler-Lehrer-Relationen ungünstig ab: Im Primarbereich kommen in Deutschland 18,8 Kinder auf eine Lehrkraft (OECD-Mittel 16,7), und im Sekundarbe-

\footnotetext{
4 Gemessen am verfügbaren Einkommen der privaten Haushalte (vgl. Statistisches Bundesamt, Fachserie 18, Reihe 1.4, Tabelle 3.4.4.1).

5 Im vergangenen Jahrzehnt ist eine Vielzahl von Einrichtungen bzw. Unternehmen aus den Kernhaushalten ausgelagert und dem nicht-staatlichen Sektor zugeordnet worden. Wie stark dieser Effekt tatsächlich war, ist kaum zu ermitteln. Allerdings waren die Einnahmen der öffentlichen Haushalte aus Gebühren in diesem Zeitraum rückläufig, was auf eine signifikante Größenordnung hindeutet, da die Gebührensätze kaum gesunken sind.
} 
reich I sind es 15,5 (OECD-Mittel 13,7). Die Betreuungsverhältnisse im Primarund Sekundarbereich I waren dabei etwas ungünstiger als 1998, dem Ausgangsjahr der Analyse. Im Sekundarbereich II liegt der Wert in Deutschland mit 14,0 Schülern pro Lehrkraft über dem OECD-Mittel $(13,0)$.

Folgt man den Angaben des Statistischen Bundesamtes, ${ }^{6}$ so haben sich die Ausgaben für Schulen und Hochschulen in Deutschland wie folgt entwickelt (Tabelle 2):

Im Hochschulbereich sind die Ausgaben gemessen am BIP nahezu unverändert geblieben; anfangs hat der Anteil leicht zugenommen, von 2004 an ist er etwas zurückgegangen. Bei den Ausgaben für allgemeinbildende und berufliche Schulen ist nach 2002 ein Rückgang zu erkennen. Der leichte Anstieg der Quote zuvor war nur vorübergehend, denn bereits in der zweiten Hälfte der 1990er Jahre war die Quote gesunken; 1995 hatte sie noch bei $2,41 \%$ gelegen.

Nicht nur im Bildungssektor, sondern in allen staatlichen Aufgabenbereichen war die Versorgung mit öffentlichen Gütern in den vergangenen Jahren rückläufig. Dies zeigt sich, wenn man die Entwicklung der staatlichen Konsumausgaben, die rund ein Viertel aller Staatsausgaben ausmachen, betrachtet (Tabelle 3). ${ }^{7}$ Selbst die öffentliche Verwaltung blieb von der Haushaltskonsolidierung nicht verschont, auch ihr Anteil am BIP hat sich verringert.

\subsection{MONETÄRE SOZIALLEISTUNGEN}

Während der Staat mit seinem Angebot an öffentlichen Gütern eher auf indirektem Wege in die Einkommensverteilung eingreift, wirkt er mit den Transferausgaben neben der Steuerpolitik - direkt auf die Verteilung ein. Bei den Transferausgaben handelt es sich insbesondere um die sogenannten monetären Sozialleistungen, also die gesetzlichen Rentenzahlungen und Pensionen, um Geldleistungen der gesetzlichen Pflegeversicherung (Pflegegeld), um Kranken- und Sterbegeld, um die Zahlungen an Arbeitslose, um die Sozialhilfe, das Wohngeld, das Kindergeld, um die Versorgung der Kriegsopfer und die Kriegsopferfürsorge und ähnliches. Die Geldleistungen der Arbeitslosen- und Sozialhilfe werden seit 2005 zum Arbeitslosengeld II zusammengefasst. Im vergangenen Jahrzehnt hat sich der Anteil der monetären Sozial-

Tabelle 2: Ausgaben für Schulen und Hochschulen - in \% des BIP -

\begin{tabular}{lccccccc|}
\hline & $\mathbf{2 0 0 0}$ & $\mathbf{2 0 0 1}$ & $\mathbf{2 0 0 2}$ & $\mathbf{2 0 0 3}$ & $\mathbf{2 0 0 4}$ & $\mathbf{2 0 0 5}$ & $\mathbf{2 0 0 6}$ \\
\hline Schulen & 2,27 & 2,27 & 2,30 & 2,29 & 2,26 & 2,26 & 2,21 \\
Hochschulen & 1,33 & 1,36 & 1,42 & 1,42 & 1,38 & 1,38 & k. A. \\
\hline $\begin{array}{l}\text { Quelle: Statistisches Bundesamt, Jahresrechnungsergebnisse der öffentlichen Haushalte } \\
\text { (Fachserie 14, Reihe 3.1), versch. Jahrgänge. }\end{array}$ & WS I MITTEILUNGEN
\end{tabular}

Tabelle 3: Staatliche Konsumausgaben nach Aufgabenbereichen

- Anteile am nominalen BIP in \% -

\begin{tabular}{lrrr|}
\hline Aufgabenbereich & $\mathbf{2 0 0 0}$ & $\mathbf{2 0 0 3}$ & $\mathbf{2 0 0 7}$ \\
\hline Allgemeine öffentliche Verwaltung & 2,43 & 2,35 & 2,27 \\
Verteidigung & 1,25 & 1,20 & 1,07 \\
Öffentliche Ordnung und Sicherheit & 1,60 & 1,63 & 1,50 \\
Wirtschaftliche Angelegenheiten & 0,40 & 0,39 & 0,26 \\
Umweltschutz, Komm. Gemeinschaftsdienste & 0,34 & 0,33 & 0,32 \\
Gesundheitswesen & 6,04 & 6,30 & 6,01 \\
Freizeit, Sport, Kultur und Religion & 0,45 & 0,41 & 0,39 \\
Bildungswesen & 3,47 & 3,55 & 3,25 \\
Soziale Sicherung & 3,01 & 3,09 & 2,92 \\
\hline Insgesamt & 19,00 & 19,26 & 17,99 \\
\hline & & W SI MITTEILUNGEN
\end{tabular}

leistungen am BIP zunächst erhöht, im Jahre 2003 erreichte er einen Wert von 19,8\%. Aufgrund der verschiedenen Eingriffe in die sozialen Sicherungssysteme ist er danach spürbar, auf 17,3\% im Jahre 2007, gesunken. Dieser Rückgang war weit stärker als in dem vorangegangenen Konjunkturzyklus und auch stärker als in früheren Aufschwungphasen (Statistisches Bundesamt 2007, Tabelle 3.4.4.7).

Im Normalfall nimmt der Anteil der Sozialleistungen im Abschwung zu und im Aufschwung ab. Zum einen hängt dies mit der antizyklischen Wirkung der Arbeitslosenversicherung zusammen, deren Ausgaben bei steigender Arbeitslosigkeit zu- und bei sinkender Arbeitslosigkeit abnehmen. Zum anderen gehen vom Rentensystem im Prinzip stabilisierende Wirkungen aus, da die Renteneinkommen den Arbeitseinkommen verzögert folgen. Doch hat die hartnäckige wirtschaftliche Stagnation die politischen Entscheidungsträger zu einer Reihe diskretionärer Maßnahmen veranlasst, mit denen die Wirkung dieser automatischen Stabilisatoren eingeschränkt wurde; auch die äußerst zurückhaltende Lohnpolitik in dieser Zeit hat dazu beigetragen. In der Folge war die Sozialleistungsquote nach 2003 stark rückläufig (Tabelle 4). Die stagnierenden Einkommen je beschäftigten Arbeitnehmer zogen stagnierende Renteneinkommen nach sich, zumal der Nachhaltigkeitsfaktor 2005 in Kraft getreten war, der den Rentenanstieg zusätzlich dämpfte. ${ }^{8}$ Zusätzliche Rentenkürzun- gen wurden durch den Riesterfaktor wirksam. ${ }^{9}$ Auch die staatlichen Pensionszahlungen und Beihilfen gingen nach 2003 anteilsmäßig merklich zurück, vor allem, weil die Gehaltsentwicklung im öffentlichen Dienst äußerst moderat war und zu-

6 Diese Daten werden auch im Rahmen des Bildungsfinanzberichts der Bund-Länder-Kommission für Bildungsplanung und Forschungsförderung verwendet. Sie sind nach dem Grundmittelkonzept abgegrenzt.

7 Die Konsumausgaben des Staates setzen sich vor allem aus den Entgelten für die Beschäftigten im öffentlichen Dienst und den Käufen von Vorleistungen zusammen. In den Vorleistungen sind die Sachaufwendungen der Gebietskörperschaften ebenso enthalten wie die als soziale Sachleistungen der gesetzlichen Krankenkassen verbuchten Medikamentenkäufe etc.

8 Im Nachhaltigkeitsfaktor drückt sich das zahlenmäßige Verhältnis von Rentnern und Beitragszahlern aus; damit wird die Entwicklung des Rentenniveaus an die demografische Entwicklung angepasst: Ein steigender Rentnerquotient führt zu Rentensenkungen. Wenn in wirtschaftlich schwierigen Zeiten die Zahl der sozialversicherungspflichtigen Beschäftigten zurückgeht, schlägt sich dies ebenfalls im Nachhaltigkeitsfaktor dämpfend nieder.

9 Durch den Riesterfaktor wurden die Renten jährlich um etwa 0,6 Prozentpunkte gedämpft. Dieser Kürzungsbetrag je Rentner entspricht etwa den zusätzlichen Belastungen der Berufstätigen für die staatlich geförderte Altersvorsorge. Die Rentner erhalten also soviel weniger, wie die Berufstätigen mehr privat vorsorgen. Der Riesterfaktor sollte eigentlich bis einschließlich 2011 abgezogen werden. Nun hat die Regierung ihn für 2008 und 2009 ausgesetzt, doch soll er in den Jahren 2012 und 2013 "nachgeholt" werden. 
Tabelle 4: Monetäre Sozialleistungen des Staates - Anteile am nominalen BIP in \% -

\begin{tabular}{lrrr} 
& $\mathbf{2 0 0 0}$ & $\mathbf{2 0 0 3}$ & $\mathbf{2 0 0 7}$ \\
\hline Deutsche Rentenversicherung & 10,06 & 10,51 & 9,57 \\
Landwirtschaftliche Alterskassen & 0,13 & 0,14 & 0,12 \\
Gesetzliche Krankenversicherung & 0,41 & 0,37 & 0,27 \\
Gesetzliche Pflegeversicherung & 0,26 & 0,23 & 0,20 \\
Gesetzliche Unfallversicherung & 0,32 & 0,32 & 0,27 \\
Arbeitslosenversicherung & 1,83 & 2,10 & 1,01 \\
Pensionen und Beihilfen des Staates & 1,71 & 2,09 & 1,94 \\
Versorgung der Kriegsopfer & 0,17 & 0,13 & 0,08 \\
Arbeitslosenhilfe & 0,64 & 0,78 & 0,97 \\
Sozialhilfe und Kriegsopferfürsorge & 0,59 & 0,66 & 0,86 \\
Kindergeld & 1,51 & 1,60 & 1,42 \\
Ausbildungsbeihilfen & 0,04 & 0,07 & 0,06 \\
Wohngeld & 0,16 & 0,23 & 0,05 \\
Übrige soziale Geldleistungen & 0,34 & 0,31 & 0,27 \\
\hline Insgesamt & 18,17 & 19,53 & 17,11 \\
\hline & & WSI MITTEILUNGEN
\end{tabular}

dem die staatlichen Beihilfen gekürzt wurden. ${ }^{10}$ Auch in der Arbeitslosenversicherung wurden Leistungen gekappt bzw. die Anspruchsvoraussetzungen für den Bezug von Leistungen verschärft, sodass die Ausgaben gesenkt werden konnten. ${ }^{11}$ Der kassenmäßige Effekt dieser Leistungskürzungen wurde von 2005 an durch die steigende Zahl an sozialversicherungspflichtigen Arbeitsplätzen verstärkt. Der Anteil der übrigen monetären Sozialleistungen am Bruttoinlandsprodukt hat sich in den letzten Jahren ebenfalls verringert.

Nicht nur die öffentlichen Güter, auch die staatlichen Sozialtransfers wurden demnach im Zuge der wirtschaftlichen Krise und als Folge der steuerreformbedingten Einnahmenausfälle merklich verringert. Damit haben die Instrumente, die zur Korrektur der marktbestimmten Einkommensverteilung dienen, erheblich an Bedeutung eingebüßt. Betroffen davon waren vor allem die unteren Einkommensschichten, die in besonderem Maße auf staatliche Sozialtransfers angewiesen sind und vom Angebot an öffentlichen Gütern profitieren. Folglich hat die staatliche Politik die äußerst ungleichgewichtige Einkommensverteilung der letzten Jahre verschärft. Dies wirft die Frage auf, ob sich die Unterund Mittelschicht noch in dem Maße wie bisher auf den Sozialstaat „verlassen“ kann (vgl. dazu auch Kronauer 2008). Durch die prozyklische Wirkung der verschiedenen Maßnahmenpakete haben die finanzpolitischen Entscheidungsträger zudem zur Schwächung der gesamtwirtschaftlichen Nachfrage und der Beschäftigung beigetragen.

\subsection{STEUERPOLITIK}

Steuerpolitische Maßnahmen sind seit jeher elementarer Bestandteil staatlicher Redistributionspolitik. Dabei kommt der Einkommensteuer besondere Bedeutung zu, da ihr ein progressiver Steuertarif zugrunde liegt. Verteilungspolitisch äußerst relevant ist die Tatsache, dass die verschiedenen Einkunftsarten teilweise sehr ungleich bei der Einkommensermittlung behandelt werden. ${ }^{12}$ Versuchen, dies zu ändern, war bisher wenig Erfolg beschieden. Stärker in den Fokus gerückt sind die verteilungspolitischen Implikationen von Mehrwertsteuererhöhungen; im Gegensatz zur Einkommensteuer, deren Sätze immer wieder gesenkt wurden, sind die Mehrwertsteuersätze mehrmals erhöht worden. Die Anhebung der Mehrwertsteuer ist ein gern genutztes Mittel, die Finanzlage des Staates zu verbessern, denn die Erhöhung wird im Gegensatz zur Einkommensteuer nur mittelbar wahrgenommen, nämlich über höhere Preise, von denen die einzelnen Einkommensgruppen sehr unterschiedlich getroffen werden. Wegen ihrer direkten Fühlbarkeit sind denn auch Einkommensteuersenkungen politisch beliebt.

\section{EINKOMMENSTEUER}

Von einschneidender Bedeutung in den letzten Jahren waren die Einkommensteuerreform 2000 in Verbindung mit einer Reform der Unternehmensbesteuerung sowie die massive Anhebung der Mehrwertsteuer 2007. Die Entlastungen bei der Einkommensteuer waren dreistufig angelegt. Eckpunk- te der Reform waren die Senkung des Spitzensteuersatzes von $53 \%$ auf $42 \%$, die Verringerung des Eingangssatzes von 25,9\% auf $15 \%$ sowie die Anhebung des Grundfreibetrages von $6.647 €$ auf $7.664 €$.

Wie immer bei Entlastungen in einem progressiven Tarifsystem zeigen sich riesige Unterschiede, wenn man die absoluten Entlastungsbeträge nach Einkommensklassen differenziert. Vergleicht man die Entlastungsbeträge der Steuerpflichtigen, die nach Grundtabelle besteuert werden, so wurden Steuerpflichtige mit einem zu versteuernden Einkommen in Höhe von $200.000 €$ pro Jahr um $15.380 €$, Einkommen in Höhe von $40.000 €$ hingegen nur um $1.554 €$ entlastet (Tabelle 5). Aussagekräftiger ist natürlich die relative Betrachtung. Auch hier zeigt sich, dass mit steigendem Einkommen die Entlastungen zunehmen: Einkommensbezieher mit einem Einkommen von $20.000 €$ wurden um 3,6\% ihres Einkommens entlastet, für Einkommen von $70.000 €$ errechnen sich $5,3 \%$ und für Einkommen in Höhe von $200.000 €$ $7,7 \%$, d.h. die hohen Einkommen wurden relativ doppelt so stark entlastet wie die niedrigen Einkommen. Längst nicht so ausgeprägt waren die Unterschiede für Familien (mit zwei Kindern). Die relativen Entlastungen weisen eher einen u-förmigen Verlauf auf. Gegenüber der Grundtabelle zeigen sich in den oberen Einkommensbereichen geringere Entlastungseffekte, während in den unteren und mittleren Einkommensbereichen die relativen Entlastungen höher waren. Dennoch bleibt,

10 Die Entwicklung der Pensionszahlungen im öffentlichen Dienst hängt neben der Zahl der Empfänger von den Tarifabschlüssen im öffentlichen Dienst ab.

11 In erster Linie sind die verschiedenen Arbeitsmarkt "reformen“ zu nennen, die u.a. die Zumutbarkeitsregeln verschärften und durch die Absenkung bzw. Streichung von staatlichen Leistungen den Druck auf Arbeitslose massiv verstärkten, auch äußerst gering bezahlte Tätigkeiten anzunehmen. Durch die Kombination von zunehmender Beschäftigungsunsicherheit - die gesamtwirtschaftlichen Rahmenbedingungen waren bei Inkrafttreten der Reformen alles andere als günstig - und dem gleichzeitigen Abbau sozialstaatlicher Absicherungen hat sich das Risiko eines sozialen Abstiegs der Beschäftigten erheblich vergrößert. Die öffentlichen Haushalte wurden durch die verschiedenen Maßnahmen freilich erheblich entlastet.

12 So besitzen Gewinneinkommensbezieher ein gewisses "Gestaltungsprivileg“, weil Ausgaben aus der privaten Konsumsphäre in die Betriebssphäre verlagert werden können. 
dass alles in allem die Senkung der Einkommensteuern die oberen Einkommensbezieher stärker begünstigte als die unteren und mittleren Einkommensschichten.

\section{MEHRWERTSTEUER}

Aus verteilungspolitischer Sicht mindestens ebenso problematisch war die kräftige Erhöhung der Mehrwertsteuersätze 2007, die mit einer Senkung der Sozialbeiträge einherging. Per saldo ergeben sich dadurch rein rechnerisch jährliche Mehreinnahmen des Staates in Höhe von 13 Mrd. €. ${ }^{13}$ Im Gegensatz zur Einkommensteuer wirkt die Mehrwertsteuer regressiv, d.h. mit steigendem Einkommen geht die Belastung zurück. Diese Regressionswirkung lässt sich durchgängig über alle Einkommensdezile der Einkommensverteilung beobachten (Bach 2005). Die Steuerbefreiungen bzw. -ermäßigungen mildern diesen Effekt, kompensieren ihn aber nicht. Die verteilungspolitische Brisanz liegt darin, dass die Sparquote mit steigendem Einkommen zunimmt. Während Haushalte mit sehr niedrigem Einkommen ihr gesamtes Einkommen ausgeben (und zur Finanzierung ihres Konsums teilweise auf andere Quellen zurückgreifen müssen), werden in den mittleren Einkommensklassen 75 bis $80 \%$ des Einkommens für Verbrauchszwecke verwendet, während im oberen Bereich nur zwei Drittel des Einkommens und weniger in den Konsum fließen.

\section{BEITRAGSSENKUNGEN IN DER SOZIALVERSICHERUNG}

Von der Reduzierung der Arbeitnehmerbeiträge zur Sozialversicherung profitierten nur die sozialversicherungspflichtigen Beschäftigten. Die Beitragssenkungen entlasteten die kleineren und mittleren Arbeitnehmereinkommen stärker als die oberen Einkommensbezieher, was vor allem an der Beitragsbemessungsgrenze der Sozialversicherungsbeiträge lag, die bei höheren Bruttoeinkommen zu einer sinkenden Belastungsquote führt (Bach 2005). Tabelle 6 gibt Auskunft über die regressive Wirkung des Maßnahmenpaketes, nämlich die Erhöhung des Regelsatzes und die Senkung des Beitragssatzes zur Sozialversicherung.

\section{UNTERNEHMENSSTEUER}

Mit der Reform der Unternehmenssteuer war die Absicht verbunden, die Bedingun-

\begin{tabular}{|c|c|c|c|c|}
\hline \multirow{2}{*}{$\begin{array}{l}\text { Zu versteuerndes } \\
\text { Einkommen } \\
\text { in Euro }\end{array}$} & \multicolumn{2}{|c|}{ Alleinstehende ohne Kinder } & \multicolumn{2}{|c|}{ Ehepaar mit 2 Kindern 1 ) } \\
\hline & $\begin{array}{c}\text { Steuerliche } \\
\text { Entlastung } \\
\text { in Euro }\end{array}$ & $\begin{array}{c}\text { in } \% \text { des zu } \\
\text { versteuernden } \\
\text { Einkommens }\end{array}$ & $\begin{array}{l}\text { Entlastung } \\
\text { in Euro2) }\end{array}$ & $\begin{array}{l}\text { in } \% \text { des zu } \\
\text { versteuernden } \\
\text { Einkommens }\end{array}$ \\
\hline 5.000 & 0 & 0,0 & 382 & 7,6 \\
\hline 10.000 & 363 & 3,6 & 382 & 3,8 \\
\hline 15.000 & 562 & 3,7 & 663 & 4,4 \\
\hline 20.000 & 728 & 3,6 & 1.109 & 5,5 \\
\hline 25.000 & 910 & 3,6 & 1.343 & 5,4 \\
\hline 30.000 & 1.109 & 3,7 & 1.506 & 5,0 \\
\hline 35.000 & 1.323 & 3,8 & 1.661 & 4,7 \\
\hline 40.000 & 1.554 & 3,9 & 1.839 & 4,6 \\
\hline 45.000 & 1.801 & 4,0 & 2.008 & 4,5 \\
\hline 50.000 & 2.064 & 4,1 & 2.203 & 4,4 \\
\hline 60.000 & 2.789 & 4,6 & 2.390 & 4,0 \\
\hline 70.000 & 3.687 & 5,3 & 2.671 & 3,8 \\
\hline 80.000 & 4.584 & 5,7 & 3.134 & 3,9 \\
\hline 90.000 & 5.481 & 6,1 & 3.628 & 4,0 \\
\hline 100.000 & 6.379 & 6,4 & 4.157 & 4,2 \\
\hline 110.000 & 7.290 & 6,6 & 4.743 & 4,3 \\
\hline 120.000 & 8.187 & 6,8 & 5.356 & 4,5 \\
\hline 130.000 & 9.085 & 7,0 & 6.182 & 4,8 \\
\hline 140.000 & 9.982 & 7,1 & 7.079 & 5,1 \\
\hline 150.000 & 10.879 & 7,3 & 7.977 & 5,3 \\
\hline 200.000 & 15.380 & 7,7 & 12.463 & 6,2 \\
\hline \multicolumn{5}{|c|}{$\begin{array}{l}\text { 1) Unter } 16 \text { Jahren. } \\
\text { 2) Der Entlastungsbetrag setzt sich aus der steuerlichen Entlastung und der Änderung der Kindervergünstigungen } \\
\text { (Kindergeld bzw. Kinderfreibetrag) zusammen. }\end{array}$} \\
\hline
\end{tabular}

gen auf der Angebotsseite zu verbessern und den Standort Deutschland für ausländische Investoren attraktiver zu gestalten. Steuersystematisch bedeutet die Reform einen radikalen Wechsel. Nicht nur wurde die Körperschaftsteuer für einbehaltene wie für ausgeschüttete Gewinne auf einheitlich $25 \%$ gesenkt, d.h. der gespaltene Steuersatz abgeschafft. Ebenso entfiel die bisherige Vollanrechnung inländischer Körperschaftsteuer auf die Körperschaft-

\begin{tabular}{|c|c|}
\hline \multicolumn{2}{|c|}{$\begin{array}{l}\text { Tabelle 6: Veränderung der } \\
\text { Steuer und Beitragsbelastung } \\
\text { in Relation zu den ausgabe- } \\
\text { fähigen Einkommen } \\
\text { - in Prozentpunkten'1) - }\end{array}$} \\
\hline Untere $5 \%$ & 1,35 \\
\hline 1. Dezil & 1,31 \\
\hline 2. Dezil & 1,11 \\
\hline 3. Dezil & 1,00 \\
\hline 4. Dezil & 0,95 \\
\hline 5. Dezil & 0,94 \\
\hline 6. Dezil & 0,90 \\
\hline 7. Dezil & 0,79 \\
\hline 8. Dezil & 0,71 \\
\hline 9. Dezil & 0,68 \\
\hline 10. Dezil & 0,64 \\
\hline Obere $5 \%$ & 0,64 \\
\hline
\end{tabular}

oder Einkommensteuer von Inländern; stattdessen wurde das sogenannte Halbeinkünfteverfahren eingeführt, was bedeutet, dass Gewinnausschüttungen bei den Anteilseignern zur Hälfte einkommensteuerpflichtig sind. Schließlich wurden die Veräußerungsgewinne steuerlich freigestellt. Zur Gegenfinanzierung wurden vor allem die Abschreibungssätze für bewegliche und unbewegliche Wirtschaftsgüter gesenkt und die steuerlichen Abschreibungszeiträume verlängert.

Gemessen an der unternehmerischen Investitionstätigkeit ist infrage zu stellen, dass die Unternehmenssteuerreform die Ziele erreicht hat, die mit ihr verfolgt worden sind. In all den Jahren nach Inkrafttreten der Reform blieb die unternehmerische Investitionstätigkeit sehr verhalten, 2002 und 2003 waren von einem deutlichen Rückgang der Investitionen geprägt. Erst seit 2004 steigen die Ausrüstungen ähnlich stark wie im vorangegangenen Aufschwung. Offensichtlich sind die Auswirkungen der verbesserten steuerlichen Standortbedin-

\footnotetext{
13 Eine genaue Quantifizierung ist nicht möglich, da bezüglich der Überwälzungsvorgänge Annahmen getroffen werden müssen. Die rechnerischen Effekte basieren auf der Annahme einer vollen Überwälzung.
} 
gungen, die seinerzeit in den Vordergrund gestellt worden waren, massiv überschätzt worden. Eine Rolle spielte dabei, dass die steuerlichen Abschreibungsbedingungen verschlechtert worden sind. Wenn durch die verschlechterten Abschreibungsbedingungen die steuerlichen Abzugsmöglichkeiten geschmälert werden, wird zudem die inländische Sachinvestition relativ zu einer Finanzanlage unattraktiver.

Gleichwohl zog die Unternehmenssteuerreform unerwartet hohe Mindereinnahmen nach sich. Im Jahre 2001 erbrachte die Körperschaftsteuer erstmals ein negatives Aufkommen. Dies war nicht nur auf die Senkung des Steuersatzes oder darauf zurückzuführen, dass sich infolge des „crash" an den Aktienmärkten und der schlechten Wirtschaftsentwicklung die Gewinnsituation der Unternehmen rapide verschlechtert hatte. Völlig unterschätzt wurde das Ausmaß des veränderten Ausschüttungsverhaltens der Unternehmen als Folge der Umstellung auf das Halbeinkünfteverfahren und der Einführung eines einheitlichen Steuersatzes für ausgeschüttete und thesaurierte Gewinne. ${ }^{14}$ Die hohen Steuerausfälle setzten die finanzpolitischen Entscheidungsträger unter dem Regime von Maastricht unter Druck, auch in der Rezession nach zusätzlichen Kürzungsmöglichkeiten auf der Ausgabenseite zu suchen.

\section{Fazit}

Das vergangene Jahrzehnt war durch gravierende Änderungen der finanzpolitischen Parameter geprägt. Bereits die finanziellen Lasten der deutschen Vereinigung und die Verpflichtungen aus dem Maastricht-Vertrag hatten den Spielraum der Finanzpolitik erheblich eingeschränkt. Poli- tisch gewollt waren signifikante Steuerentlastungen. Mit ihnen war die Hoffnung verbunden, dass die deutschen Unternehmen eine noch bessere Position im internationalen Standortwettbewerb erreichen würden. Tatsächlich konnte der Außenbeitrag stetig ausgeweitet werden; ob die Steuerpolitik hierauf nennenswerten Einfluss hatte, ist allerdings zweifelhaft. Tatsache ist auch, dass dennoch die Wirtschaft lange Zeit stagnierte, weil die Binnennachfrage schwach blieb. Dies wiederum lässt sich zum Teil mit der Ausrichtung der Finanzpolitik begründen. Nicht nur hat der Zwang, die staatlichen Defizite zu begrenzen, konjunkturelles „Atmen“ der öffentlichen Haushalte behindert. Auch zeitigten die Steuerentlastungen trotz ihres Umfangs konjunkturell wenig Erfolg: Die Investitionstätigkeit der Unternehmen war in den ersten Jahren nach Inkrafttreten der Steuerreform sogar rückläufig, und die privaten Haushalte erhöhten aus Unsicherheit ihre Sparquote. Insofern waren die Instrumente zur Befeuerung der wirtschaftlichen Entwicklung in jener Zeit nicht adäquat gewählt. Konjunkturell erfolgreicher wäre eine Strategie gewesen, die stärker die unteren und mittleren Einkommensschichten begünstigt hätte. Auch wäre eine Infrastrukturoffensive dringend vonnöten gewesen. Sie hätte kurzfristig Nachfrage und Beschäftigung geschaffen, langfristig wären die wirklichen Wachstumsengpässe in Deutschland, insbesondere im Bildungssektor, beseitigt worden.

Als problematisch sind auch die verteilungspolitischen Implikationen der finanzpolitischen Strategie zu werten. Die Entwicklung der Markteinkommen im letzten Jahrzehnt hat die Schere deutlich zugunsten der oberen Einkommensklassen geöffnet. Offensichtlich war es nicht Ziel der staatlichen Umverteilungspolitik, diese Diskrepanz zumindest im Ansatz zu korrigieren. Das Gegenteil war der Fall - auf allen staatlichen Aktionsfeldern waren die Weichen so gestellt, dass die disparate marktmäßige Entwicklung zugunsten der oberen Einkommensschichten verstärkt wurde. Damit schwamm Deutschland gegen den internationalen Trend. Zwar wurden in vielen anderen Ländern im gleichen Zeitraum ebenfalls die Steuerlasten gesenkt, doch profitierten davon oftmals insbesondere die unteren Einkommensschichten (OECD 2008).

Zweifelsfrei war das letzte Jahrzehnt das Jahrzehnt der Angebotspolitik. Die Politik der Lohnzurückhaltung war begleitet von einer Politik der staatlichen Zurückhaltung: Die Anteile der Staatsausgaben am BIP wurden trotz schwacher Wirtschaftsentwicklung zurückgeführt, und auch die Abgabenquote wurde gesenkt. Zumindest kurzfristig hat all dies die Wirtschaft erheblich geschwächt. Und auch die staatliche Finanzierungsposition hatte sich über lange Jahre nicht verbessert. Doch liegt die Stärke der Angebotspolitik in der langfristigen Verheißung der Problemlösung. Somit bleibt zu hoffen, dass wir die Früchte der Politik doch noch ernten können, sich die jüngsten Erfolge als von dauerhafterer Natur erweisen und der Staat wieder über mehr finanzielle Möglichkeiten verfügt, die inzwischen aufgelaufenen Probleme (Infrastrukturpolitik, Verteilungspolitik) zu lösen.

\footnotetext{
14 Den Kapitalgesellschaften war es bis Ende 2001 gestattet, zuvor einbehaltene Gewinne, die in den Bilanzen mit $45 \%$ bis 1998 und $40 \%$ ab 1999 versteuert waren, an die Anteilseigner auszuschütten und zugleich einen Erstattungsanspruch in Höhe der Differenz zwischen Einbehaltungssatz (45\%) und damaligem Ausschüttungssatz (30\%) geltend zu machen. Wenn Unternehmen nachträglich thesaurierte Gewinne an die Anteilseigner ausschütteten, erhielten sie die Differenz zwischen Thesaurierungs- und Ausschüttungssatz von den Finanzämtern zurück.
} 
Allmendinger, J./Helbig, M. (2008): Zur Notwendigkeit von Bildungsreformen, in: WSI-Mitteilungen 7, S. 394-399

Bach, S. (2005): Koalitionsvertrag: Belastungen durch Mehrwertsteuererhöhung werden nur zum Teil durch Senkung der Sozialbeiträge kompensiert, in: Wochenbericht des DIW 47

Böttcher, W. (2005): Soziale Auslese und Bildungsreform, in: Aus Politik und Zeitgeschichte 12, S. 7-13

Bofinger, P. (2008): Das Jahrzehnt der Entstaatlichung, in: WSI-Mitteilungen 7, S. 351-357

Kronauer, M. (2008): Verunsicherte Mitte, gespaltene Gesellschaft?,

in: WSI-Mitteilungen 7, S. 372-378

Matzner, E. (1982): Der Wohlfahrtsstaat von morgen, Frankfurt/New York OECD (2007): Bildung auf einen Blick. OECD Indikatoren, Paris OECD (2008): Taxing Wages 2006-2007, Paris
Rothschild, K. W. (1986): Der Wechsel vom keynesianischen zum neoklassischen Paradigma in der neueren Wirtschaftspolitik. Versuch einer soziologisch-historischen Einordnung, in: Krupp, H.-J./Rohwer, B./Rothschild K. W. (Hrsg.): Wege zur Vollbeschäftigung, Freiburg i.Br., S. 107123

Schettkat, R. (2002): Bildung und Wirtschaftswachstum, in: Mitteilungen aus der Arbeitsmarkt- und Berufsforschung 4, S. 616-627

Statistisches Bundesamt (versch. Jg.): Jahresrechnungsergebnisse der öffentlichen Haushalte (Fachserie 14, Reihe 3.1)

Statistisches Bundesamt (2007): Fachserie 18/Reihe 1.4, Volkswirtschaftliche Gesamtrechnungen

Vesper, D. (2007): Vom Stabilitäts- und Wachstumsgesetz zum Europäischen Stabilitäts- und Wachstumspakt; Wiso-Diskurs der Friedrich-EbertStiftung, Bonn 